\title{
CÉDRIC RICHET
}

BASTIEN SOULÉ

CHRISTOPHE DURAND

Université de Caen Basse-Normandie

\section{L'approche économique de la gestion déléguée en question}

\section{Le cas des complexes aquatiques}

Une approche économique de la délégation de service public permet d'appréhender les coûts de transaction entre agents publics et privés; démarche intéressante pour toute collectivité souhaitant maîtriser un tel partenariat, ou y renoncer. Toutefois, à cet égard, les pistes classiquement proposées peuvent s'avérer inadéquates, ce que révèle notamment une immersion au sein d'une société spécialisée dans la gestion déléguée des complexes aquatiques. 
$\mathrm{P}$ armi différentes formes contractuelles de partenariats public-privé (PPP), la délégation de service public (DSP) occupe une place importante en France. Cette procédure accompagne en effet les démarches de décentralisation et de responsabilisation du management public (Auby, 1995). À l'instar de nombreux secteurs d'activités, de plus en plus d'équipements sportifs et culturels font l'objet d'une gestion déléguée. Aujourd'hui, près de deux cents piscines et centres aquatiques publics sont ainsi confiés aux soins de gestionnaires privés ${ }^{1}$ contre une quarantaine seulement au tournant du siècle dernier ${ }^{2}$.

Des litiges et des échecs de gestion sont toutefois apparus ces dernières années, relativisant le succès espéré de la délégation des complexes aquatiques "sports et loisirs » (CASL). On peut dès lors s'interroger sur l'adaptation de ce mode de gestion à un secteur qui admet traditionnellement la régie (on comptabilise plus de trois mille piscines publiques en France $^{3}$ ). Certains élus sont ainsi confrontés à un dilemme s'inscrivant au cœur d'une problématique économique classique: make or buy. Un choix qui s'avère d'autant plus difficile que, dans sa logique, la DSP soulève la question de la qualité du service fourni aux différents usagers. Par ailleurs, et au-delà des interrogations sur le principe même de l'externalisation, les chambres régionales des comptes ont pu observer de multiples difficultés allant du contentieux lourd à la faillite de certains prestataires.

En empruntant aux courants contractuels issus de l'économie de la firme (théorie des coûts de transaction, théorie de l'agence et théorie des contrats incomplets ${ }^{4}$ ), nous examinerons ici les possibles interprétations des difficultés de la gestion déléguée des CASL, et ce sous l'angle particulier des transactions entre délégants et délégataires. Cette connaissance peut en effet affiner le débat faire/faire faire dans ce secteur. Au préalable, nous décrivons le contexte, ce qui nous permet de mieux saisir les problèmes que posent concrètement l'incomplétude contractuelle puis l'opportunisme des agents impliqués dans une mission d'intérêt général à caractère sportif et ludique. Dans un dernier temps, nous développons les avantages et limites des solutions classiquement proposées pour contourner ces difficultés.

\section{MÉTHODOLOGIE}

Cette étude s'appuie sur des observations réalisées au cours d'une mission de deux ans au sein d'une société spécialisée dans la gestion déléguée des loisirs sportifs. Dix complexes aquatiques en affermage ont été retenus pour constituer un échantillon intéressant à suivre (voir tableau 1). La consultation des cahiers des charges, des contrats de DSP et des rapports d'activités a permis de constituer une première base de données. Des entretiens semi-directifs menés auprès du directeur général de la société, du responsable des études, du chef de projet d'ouverture des sites, des directeurs d'exploitation régionaux et des directeurs de centre ont complété ce dispositif.

1. Patrick Bayeux, «Piscines publiques. Quels modes de gestion? Avantages et inconvénients », Atelier du

21 novembre 2007 au Salon des maires et des collectivités locales.

2. La Lettre de l'Économie du Sport n ${ }^{\circ} 578$ du 4 juillet 2001.

3. Données du Recensement des équipements sportifs du ministère de la Jeunesse, des Sports et de la Vie associative.

4. Pour une synthèse voir Baudry (2003). 


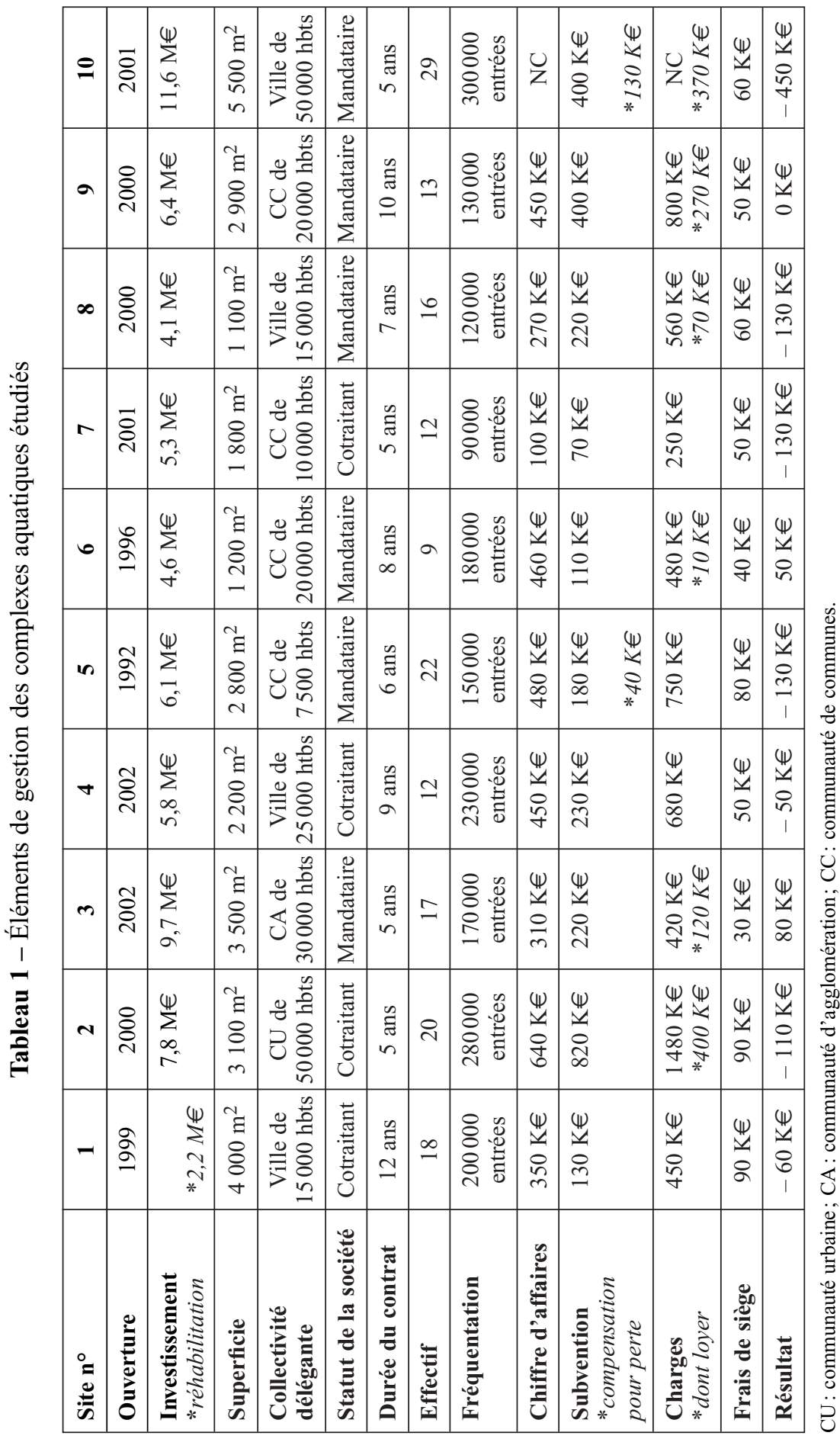




\section{I - UN CONTEXTE D'ACTION PUBLIQUE AMBIGU}

Plusieurs aspects caractérisent la gestion déléguée des CASL. La nature composite du service brouille la définition des critères de gestion. S'entrelacent par ailleurs les enjeux d'une activité d'intérêt général et d'un montage juridique particulier. Enfin des structures fortement hétérogènes en termes de poids financier et de métier investissent le marché.

\section{Un service aux multiples facettes}

Les CASL sont le résultat d'une hybridation entre les piscines traditionnelles et les parcs aquatiques (Bessy, 2002). Conçus le plus souvent dans une perspective intercommunale, ces équipements innovants et structurants allient prestations sportives et services de loisir. L'objectif social du service est de répondre à la diversité des besoins (apprentissage, compétition, loisir) et de satisfaire différents publics (scolaires, adhérents, familles). Les CASL présentent dès lors une offre relativement étendue: bassins sportifs mais aussi ludiques avec boules à vagues, toboggans, jets hydromassants, etc. Par ailleurs, l'objectif économique qui est d'atteindre le petit équilibre financier passe également par la captation d'une clientèle solvable. Ainsi, la maitrise d'ouvrage prévoit généralement l'adjonction de services commerciaux annexes: remise en forme, cafétéria, bowling, squash, etc. Les CASL sont donc des espaces multifonctions et multiactivités qui dépassent assez largement le cadre du service public envisagé par le législateur.

Or, cette variété des usages complique la définition du service public et surtout l'établissement de critères objectifs d'évaluation et de comparaison. Selon des experts des collectivités publiques, il existe même une réelle difficulté à définir les priorités, la conciliation de logiques a priori contradictoires (les sports et les loisirs) étant un obstacle à franchir (Bayeux et Cranga, 2002). L'exploitation des CASL pose en plus des questions de sécurité, de salubrité et de santé publique qui ne peuvent être résolues sans un solide savoir-faire technique et sans un suivi rigoureux.

\section{Des enjeux croisés}

Les piscines «nouvelle génération » cristallisent des intérêts et des points de vue différents, de sorte que plusieurs enjeux peuvent se télescoper (Bessy, 2002). Afin d'obtenir des profits symboliques et électoraux, les décideurs locaux sont notamment tentés de privilégier des équipements modernes, ludiques et touristiques, susceptibles de renvoyer une image dynamique de leur collectivité. A contrario, pour défendre la pratique de la natation selon les normes de la compétition, des acteurs sportifs institutionnels manifestent quelquefois des résistances à l'innovation, se constituent en groupes de pression et exercent un lobby sportif (cas du site $\mathrm{n}^{\circ} 1$ ). Les élus ont alors comme dernier levier l'arbitrage des temps de bassin et des lignes d'eau (volumes et horaires).

La volonté politique locale peut être également contrariée par la lourdeur des équipements qui nécessitent d'importants investissements financiers et humains. Or, la mise en place de PPP permet normalement de répartir les risques de gestion: les pouvoirs publics se chargent des décisions politiques tandis que les opérateurs privés assument des risques d'exploitation techniques et commerciaux. Mais cette complémentarité 
n'est qu'apparente: la seule perception des recettes sur les usagers ne permet généralement pas au délégataire d'atteindre le seuil de rentabilité (voir tableau). Le compte d'exploitation ne peut effectivement s'équilibrer sans la subvention négociée en début de contrat avec la collectivité. Ce système compensatoire se justifie par des prestations hors marché dont l'accueil des scolaires et des clubs ainsi que le tarif social imposé par la collectivité pour l'accueil du grand public. C'est un levier central de la négociation et un élément déterminant du contrat.

\section{Un marché encore immature}

Pour l'essentiel, les délégataires privés des complexes aquatiques sont des sociétés spécialisées dans l'animation sportive et de loisir: Vert Marine, Récréa, Carilis, Segap, Prestalis, Gesclub, Forest Hill. Il s'agit de structures commerciales récentes, le marché étant ouvert depuis le début des années 1990. Certaines d'entre elles sont d'ailleurs encore faiblement dotées en capital. En marge de cet oligopole, figurent des associations telles l'Union des centres de plein air (UCPA) et quelques sociétés d'économie mixte.

Ces opérateurs privés doivent très souvent s'associer, pour des raisons techniques, à des prestataires spécialisés dans le domaine de la gestion de l'eau, de la chaufferie et de la maintenance. À l'exception d'entreprises familiales implantées localement, il s'agit de filiales de grands groupes industriels se disputant le marché de l'énergie en France et dans le monde: Dalkia France (Veolia, EDF), Elyo (Suez, Lyonnaise des eaux), Saur (PAI, Bouygues), Cofathec services (GDF). Ces grands groupes détiennent quelquefois le contrat de DSP à la place des sociétés spécialisées dans l'animation. Ces dernières, qui ne peuvent afficher une aussi grande solidité financière auprès des collectivités locales, sont dès lors reléguées au statut de cotraitant (voir tableau). La légitimité économique est en effet un critère important pour remporter le marché.

Ce dernier point nous amène à considérer les difficultés de la gestion déléguée des CASL sur un plan économique. Selon un rapport de l'Institut de la gestion déléguée (IGD, 2005), améliorer la connaissance des coûts de délégation est une attente forte des collectivités. En raison de la rationalité limitée des acteurs, espérer parvenir localement à une connaissance parfaite et contingente de tels coûts semblent en effet difficile.

\section{II - UNE RATIONALITÉ LIMITÉE SOURCE D'INCOMPLÉTUDE CONTRACTUELLE}

Dans le cadre de la théorie des coûts de transaction, identifier les contingences futures et spécifier ex ante les adaptations nécessaires est très coûteux, voire techniquement impossible. Dans un environnement complexe et incertain, l'agent économique dispose en effet d'une information incomplète. De surcroît, il possède une capacité cognitive limitée qui l'oblige à restreindre sa vision des possibles. Délégants et délégataires ne sont donc pas totalement en mesure de déterminer précisément ex ante tous les paramètres de gestion (fréquentation, niveau des charges, etc.) ni de fixer exactement le montant de la subvention qui convient pour un équilibre, encore moins de prévoir les effets d'une éventuelle concurrence locale à venir. L'incomplétude contractuelle et les litiges qui peuvent en résulter ex post génèrent ainsi des coûts de 
transaction correspondant aux coûts des renégociations, voire des procès. En outre, le contexte législatif et la faiblesse économique des exploitants spécialisés dans les loisirs sportifs (alors incapables d'apporter de réelles garanties financières ou de prendre en charge les surcoûts) compliquent la donne. L'enjeu concernant la récupération de la TVA afférente aux investissements réalisés par la collectivité est, à ce sujet, assez révélateur.

\section{Comment récupérer la TVA sur les investissements?}

Dans le cas d'une délégation, la TVA ayant grevé la réalisation d'un CASL peut être récupérée via le délégataire selon une procédure de « transfert de droit à déduction ». En attendant que l'exploitant déclare la TVA, l'autorité publique demande généralement une caution à son délégataire; chose délicate dans le secteur investigué sachant que les entreprises spécialisées dans l'animation des loisirs sportifs ne disposent pas toutes d'assises financières importantes. $\mathrm{Ne}$ pouvant ainsi compter sur l'avance de fonds bancaires, une collectivité a stipulé dans son contrat de DSP l'acquittement de la TVA due par les services fiscaux sur son compte public sans passer par celui du délégataire (cas du site $n^{\circ} 3$ ). Elle n'imaginait cependant pas qu'une telle procédure dérogeait aux règles de droit commun concernant les obligations déclaratives des redevables de la TVA, ni qu'elle connaîtrait des déboires pour récupérer cette taxe.

\section{Une renégociation souvent difficile et inégale}

$\mathrm{Ne}$ pouvant prévoir tous les événements futurs et n'entendant pas pour autant subir les conséquences d'événements non antici- pés ou sous-estimés, les contractants sont amenés à procéder à d'éventuelles renégociations. Mais, revenir sur les clauses du contrat de DSP n'est pas chose simple, car à cette occasion, un nouveau rapport de force s'établit entre délégant et délégataire. Des tractations portant sur le montant de la subvention d'équilibre ou des besoins non prévus (dispositif de surveillance par exemple) ont parfois abouti à de coûteux procès (cas du site $n^{\circ} 4$ ). Le flou qui entoure la mission de service public dans les CASL est souvent à l'origine de tels contentieux. En raison de l'asymétrie que le cadre institutionnel instaure entre le délégant et le délégataire (Ménard et Saussier, 2003), l'un et l'autre n'ont pourtant pas le même pouvoir de négociation. L'autorité délégante dispose en effet de prérogatives importantes, dont le droit de modification unilatéral du contrat. Par contre, le délégataire assure la gestion " à ses risques et périls » et doit notamment supporter les conséquences d'un éventuel déficit. Certes, un avenant peut être conclu et, selon l'IGD (2005), cela semble être fréquemment le cas en matière de services culturels et sportifs. Mais quand le déséquilibre constaté provient des prévisions financières de l'entreprise ou du déroulement du contrat, le délégataire n'a, en principe, aucun droit à entrer en négociation. Il ne peut obtenir un avenant qu'à deux conditions : 1) que la collectivité accepte de négocier et 2) que ce changement ne porte pas atteinte à l'égalité initiale des concurrents.

Si la question de remédier ou non à un déséquilibre financier se pose finalement avec autant d'acuité, c'est que celle concernant l'information obtenue sur le délégataire n'a pas été résolue. En d'autres termes, la collectivité n'est pas totalement en mesure de 
savoir si l'exploitant dispose de la capacité suffisante et/ou de la volonté nécessaire pour surmonter le coût du service public. Un autre type d'asymétrie existe donc entre délégant et délégataire.

\section{III - UNE ASYMÉTRIE D'INFORMATION SOURCE D'OPPORTUNISME}

Bien qu'elle postule la rationalité substantive des contractants, la théorie de l'agence peut, cette fois, éclairer le mécanisme contractuel des PPP. En effet, la relation entre partenaires publics et privés est fondamentalement de type principal-agent (Marty et al., 2006). La difficulté d'une telle relation réside dans son déséquilibre: toute collectivité (le principal) est soumise à une asymétrie d'information vis-à-vis des entreprises sur le marché (les agents). D'une part, l'autorité publique ne peut avec certitude distinguer, ex ante, les firmes performantes des autres, et d'autre part, elle ne peut que difficilement déterminer, ex post, si la firme choisie met en œuvre les efforts nécessaires pour réduire ses coûts.

\section{Le cercle vicieux de la « sélection adverse »}

Le premier type d'asymétrie informationnelle entraîne un phénomène « d'antisélection » qui semble manifestement tirer le marché de la DSP des CASL vers le bas. En effet, un prestataire désireux de remporter le marché peut tricher sur ses véritables qualités et compétences; d'autant que la nature du service dans le secteur des loisirs sportifs se prête assez mal à l'établissement de critères objectifs de performance. De fait, en se banalisant, ce comportement opportuniste précontractuel fausse le mar- ché: un prix élevé de prestations n'est pas forcément un signal de qualité fiable aux yeux d'élus. Ceci les encourage ainsi à maintenir une pratique courante qui consiste à rechercher le « moins-disant » au lieu du « mieux-disant ».

Ce problème de « sélection adverse » est assez pernicieux car certaines sociétés ont un temps adopté une stratégie marketing répondant à cette logique économique. Se sentant obligés d'accepter les règles $d u$ marché pour décrocher des contrats, des candidats proposaient des charges prévisionnelles dangereusement optimistes par rapport à la réalité d'une exploitation. Une fois sur le terrain, le délégataire désigné n'était pas toujours en mesure d'équilibrer ses comptes ni de remplir ses engagements (phénomène appelé la «malédiction du vainqueur »). Certains échecs de gestion des CASL sont directement imputables à cette cause (cas du site $n^{\circ} 10$ ). D'ailleurs, le déficit d'exploitation observé pour la plupart des sites témoigne en partie de ce déséquilibre (voir tableau).

\section{Un risque de « capture » limité mais bien réel}

Le second type d'asymétrie informationnelle fait naître une situation de " risque moral », source d'opportunisme postcontractuel cette fois, puisque le délégant peut être confronté à l'aléa de comportement du délégataire pendant l'exécution du contrat. Ce dernier peut revenir sur des promesses qui lui ont permis de remporter l'appel d'offres (quota d'événements sportifs à organiser non respecté), pratiquer des tarifs supérieurs au prix proposé (pour les activités de remise en forme notamment), rogner sur la qualité du service (conséquence d'une politique de gestion du personnel en 
flux tendu), ne pas renouveler certains investissements (mobiliers et matériels pédagogique par exemple) ou même chercher à renégocier le contrat dans les plus brefs délais.

Le délégataire peut d'autant plus facilement imposer ses conditions que son éventuel remplacement est long et coûteux. De plus, le délégant doit, bon an mal an, garantir la continuité du service public. L'autorité publique peut donc assez facilement se retrouver coincé dans une relation de dépendance. Toutefois, dans le cas des CASL, le risque de hold-up est limité par la structure oligopolistique du marché et le faible degré de spécificité des actifs engagés dans la transaction qui font qu'un prestataire de loisirs sportifs n'est pas irremplaçable. Par ailleurs, un opérateur privé qui gère de nombreux sites cherchera en général à préserver sa réputation.

Si l'on intègre maintenant la théorie des contrats incomplets, laquelle considère comme seule source sérieuse d'incomplétude l'incapacité d'une tierce partie de vérifier l'information détenue par les contractants, il est indispensable de chercher à gérer au mieux rationalité limitée et asymétries informationnelles. Cela signifie donc, en pratique, mettre en place des dispositifs permettant d'y remédier. La dernière partie de l'article vise à interroger deux procédés classiques à l'aune de cette théorie.

\section{IV - LIMITES DES OPTIONS CLASSIQUEMENT PRÉCONISÉES POUR RENFORCER LES CONTRATS}

Pour répondre aux besoins d'adaptabilité et de sécurité des contrats, deux formes principales de coordination, souvent citées dans les relations d'échange économique
(Baudry, 2005), sont appliquées à la DSP des CASL: l'autorité et l'incitation. Mais le contexte défini précédemment ainsi que le jeu des acteurs en présence limitent, dans les faits, l'efficacité de tels dispositifs.

\section{L'autorité : une mesure délicate à mettre en œuvre}

L'autorité, " entendue comme transfert contraint du pouvoir de décision d'un agent envers un autre agent » (Baudry, 2005, p. 39) peut remédier partiellement à la rationalité limitée des contractants et limiter le déploiement de comportements opportunistes.

Selon la théorie des contrats incomplets, le choix des actions à entreprendre en cas d'imprévu revient à la partie qui détient les actifs les plus importants, en l'occurrence la collectivité qui, en qualité de maître d'ouvrage, récupère le bâti à la fin du contrat. Le délégant possède donc un « droit résiduel de contrôle » sur cet actif. D'ailleurs, lorsqu'il s'agit de gérer l'incomplétude d'un contrat, l'autorité publique a traditionnellement recours à une logique administrative: elle s'arroge le droit de décider ponctuellement ce qu'il convient de faire (application en droit de la théorie de l'indécision). Lorsque ses prescriptions ont pour conséquence d'alourdir les conditions d'exécution financières du contrat, elle octroie à son prestataire une indemnité compensatrice (application en droit de la théorie du fait du prince). En cas d'aléas, la jurisprudence du Conseil d'État reconnaît en effet un droit à l'équilibre économique du contrat pour le délégataire. Par exemple, en raison des difficultés techniques liées à la conception des piscines (étanchéité, sécurité), les exploitants sont souvent obligés d'anticiper certains travaux de rénova- 
tion. Les collectivités, sous couvert de la garantie décennale, versent alors une aide financière visant à surmonter les déficits d'exploitation dus aux arrêts techniques (cas du site $\mathrm{n}^{\circ}$ 5). Mais quid des actifs humains " détenus » par l'opérateur privé? Que peut faire une collectivité pour empêcher l'éventuelle rupture du service public lorsque le personnel salarié menace de faire grève (cas du site $\left.n^{\circ} 2\right)$ ?

Pour contraindre le délégant à l'honnêteté, le contrôle est une solution courante mais l'application de sanctions dissuasives est délicate compte tenu du contexte évoqué. En vertu d'un principe propre aux contrats administratifs, l'autorité publique peut en effet prononcer la déchéance du délégataire en cas de faute lourde. Cette mesure se traduit par la résiliation unilatérale du contrat sans avoir besoin de saisir le juge administratif. La personne publique peut également infliger à son prestataire des pénalités financières en cas de non-réalisation du service. Mais de telles sanctions font courir un risque important: celui de déclencher une crise de solvabilité chez un contractant déjà en difficulté. Or, comme nous l'avons vu, ce risque est particulièrement élevé pour des petits prestataires de services sportifs et de loisirs. De surcroît, la puissance publique se retrouve elle-même dans une position délicate quand elle doit, en dernier recours, assurer la continuité du service public alors qu'elle ne dispose pas des ressources et des compétences nécessaires. Ajoutons que dans le secteur des CASL, les délégants sont souvent de petites structures intercommunales dépourvues de services ou de cadres sportifs (voir tableau). Aussi, la mise en régie provisoire ou la déchéance du délégataire ne constitue guère un système de menaces « crédibles »
(Ménard et Saussier, 2003). Par ailleurs, l'activation des clauses de pénalités ne va pas de soi. Ces modalités organisationnelles coercitives n'empêchent pas d'éventuels comportements opportunistes (cas du site $\mathrm{n}^{\circ} 5$ ), les actions n'étant pas totalement observables et/ou mesurables par des tiers (Farès et Saussier, 2002). Prévoir des clauses incitatives à la performance est-il alors préférable?

\section{L'incitation : un mécanisme toujours fragile}

Dans le cadre d'une relation d'agence, l'incitation peut être considérée comme un processus qui « amène un agent à révéler l'information qu'il détient puis le pousse à agir en conformité avec les objectifs fixés par le principal sur la base de cette information » (Ménard, 2004, p. 60). Ex ante, le mécanisme d'appel d'offres dans la procédure de DSP place les candidats en position de concurrents. Théoriquement, cette situation les oblige à révéler leur information, c'està-dire à montrer leur capacité réelle à exécuter le service et le prix qu'ils sont prêts à sacrifier pour y parvenir. Mais nous avons déjà vu les limites du dispositif: la tricherie n'est pas exclue, surtout lorsque le service est complexe et sujet à une forte incertitude. En pareille situation, une procédure négociée est d'ailleurs préférable au simple appel d'offres (Marty et al., 2006). Demander à choisir dans un menu de contrats différents, l'un privilégiant l'efficacité productive grâce à une prime fixe (contrat fixed price) et l'autre l'efficacité allocative via le remboursement des coûts (contrat cost plus), peut être une parade (Cartelier, 2007).

Ex post, une solution courante existe pour inciter une firme engagée dans un partena- 
riat à coopérer: le partage de la rente. Dans un contrat de DSP, le délégant attribue normalement au délégataire le statut de créancier résiduel. Le premier reste le propriétaire des actifs et le second exploite le service public en récupérant la rente générée par l'activité. Mais des clauses contractuelles d'intéressement prévoient aussi le partage des excédents entre le délégataire et la collectivité à partir d'un certain niveau de résultat d'exploitation. Pour conserver l'exclusivité des gains, le délégataire peut cependant gonfler le montant des charges, en injectant notamment des frais supplémentaires de structure générale dans le compte d'exploitation de la piscine, voire en majorant le coût du personnel. Ce procédé est en général un bon moyen de faire apparaître un déficit plus important afin de réclamer une subvention d'équilibre plus conséquente lors d'une prochaine renégociation (cas du site $\mathrm{n}^{\circ} 1$ ). Désormais, pour plus de transparence dans les comptes, des collectivités exigent la création de sociétés ad hoc et/ou stipulent le partage de la rente sur la base du chiffre d'affaires réalisé.

Comme les standards techniques imposés contractuellement ne suffisent quelquefois pas, des économistes préconisent le recours à une agence de réglementation complémentaire, se ralliant de fait au postulat de la théorie des contrats incomplets (Saussier et Yvrande-Billon, 2007).

\section{CONCLUSION}

Une approche économique de la gestion déléguée des CASL, mettant en exergue l'incomplétude contractuelle et l'opportunisme des agents, enrichit sans conteste notre connaissance des difficultés des PPP dans ce secteur. Elle peut de surcroît affiner le débat faire/faire faire entre une régie et une délégation en dépassant le clivage politique gauche/droite ou idéologique public/ privé. En prenant appui sur des facteurs économiques, il semblerait ainsi possible de préciser les cas où une collectivité locale ne doit pas déléguer le service public. Pour Ménard et Saussier (2003), la régie publique est par exemple préférable lorsque les coûts de transaction sont trop élevés eu égard aux gains à espérer en termes de coûts de production et lorsque le service attendu, notamment en terme de qualité, n'est pas parfaitement contractualisable...

Néanmoins, les exemples tirés de nos observations incitent à la prudence. Tout d'abord, en raison d'hypothèses et de préoccupations différentes, les approches contractuelles mobilisées peuvent être, sur un plan normatif, fondamentalement divergentes. Par ailleurs, ces théories, trop statiques, ne prennent pas en compte la capacité des agents à évoluer par routines, apprentissages et acquisitions de connaissances. Ensuite, une distinction doit être opérée entre différentes formes de contrat comme la concession de service public, l'affermage et la régie intéressée. Le risque financier et le niveau de responsabilités partagés entre délégant et délégataire varient fortement selon les cas. Enfin, comme nous avons pu le constater, les prescriptions formulées, pour peu qu'elles soient détachées du contexte et des logiques d'action propres au secteur concerné, peuvent s'avérer inappropriées.

Les courants contractuels récents tentent d'y remédier via des propositions alternatives et contingentes. La mise en place d'interfaces, c'est-à-dire de « cellules » comprenant des représentants de la personne publique, de la personne privée et des usa- 
gers, est une possibilité. De tels comités paritaires de suivi peuvent être prévus dans le contrat de DSP pour diligenter des expertises contradictoires, formuler des remarques, engager des procédures d'évaluation, de médiation ou de négociation. Ce mode non juridictionnel de règlement des litiges ne permet cependant pas d'aller jusqu'à l'arbitrage, rôle pour l'instant dévolu au juge du contrat.

\section{Bibliographie}

Auby J.-F., La délégation de service public, PUF, Paris, 1995.

Baudry B., Économie de la firme, La Découverte, Paris, 2003.

Baudry B., L'économie des relations interentreprises, La Découverte, Paris, 2005.

Bayeux P., Cranga J.-C., « L'intercommunalité, niveau de gestion d'équipements aquatiques de plus en plus lourds », Espaces, n 194, 2002, p. 37-38.

Bessy O., "L'innovation dans les piscines publiques », Les espaces sportifs innovants. L'innovation dans les équipements, Bessy O., Hillairet D., tome 1, Presses Universitaires du Sport, Voiron, 2002, p. 179-237.

Cartelier L., «Production et régulation des services en réseau: l'évolution de l'analyse économique ", Cahiers français, $\mathrm{n}^{\circ}$ 339, La Documentation française, 2007, p. 51-57.

Fares M., Saussier S., "Coûts de transaction et contrats incomplets », Revue française d'économie, vol. 16, n 3, 2002, p. 193-230.

Institut de la Gestion Déléguée, Quelle compétition pour l'amélioration du service public?, La Documentation française, Paris, 2005.

Marty F., Trosa S., Voisin A., Les partenariats public-privé, La Découverte, Paris, 2006.

Ménard C., L'économie des organisations, La Découverte, Paris, 2004.

Ménard C., Saussier S., «La délégation de service public, un mode organisationnel efficace? Le cas de la distribution d'eau en France », Économie Publique, vol. 12, n ${ }^{\circ}, 2003$, p. $99-129$.

Saussier S., Yvrande-Billon A., Économie des coûts de transaction, La Découverte, Paris, 2007. 